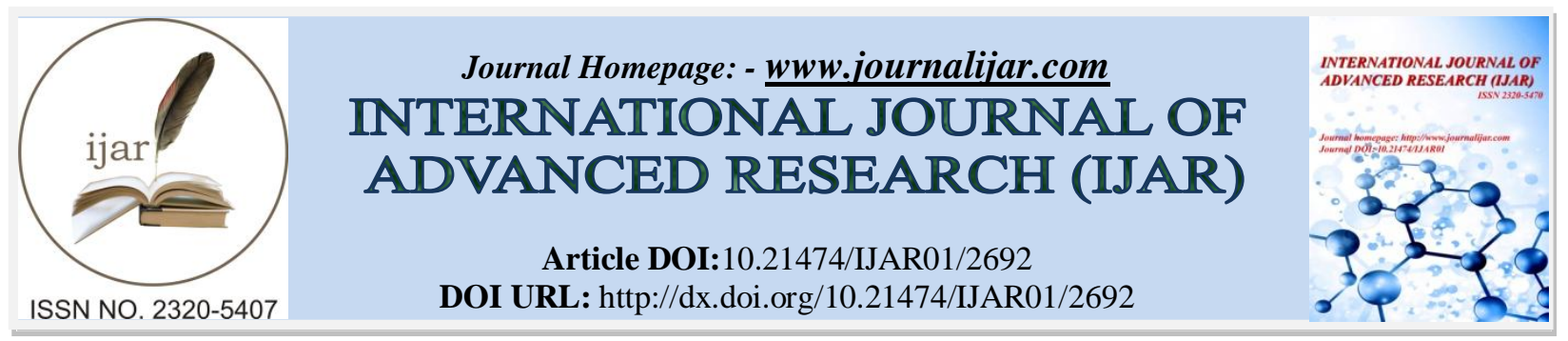

RESEARCH ARTICLE

\title{
HEPATOTOXICITY INDUCED BY GIBBERELLIC ACID (GA3) IN ADULT MALE ALBINO RATS.
}

Hanan M. Abd El-Latief.

Zoology Department, Women's College for Arts, Science and Education, Ain Shams University, Cairo, Egypt.

\section{Manuscript Info}

Manuscript History

Received: 31 October 2016

Final Accepted: 01 December 2016

Published: December 2016

Key words:-

Hepatoxicity; Gibberellic Acid; Plant

Growth Regulators; Ultrastructure.

\section{Abstract}

The present investigation aims to evaluate the effects of gibberellic acid (GA3) onthe histology and ultrastructure of the liver of rats and to assess the possibility of recovery after GA3 withdrawal.

Male albino rats were equally divided into five groups. Group I served as control. Groups II\&III received GA3 daily in drinking water (100 and $200 \mathrm{ppm}$, respectively) for 8 weeks. Groups IV\&V received 100 and 200 ppm GA3; then were left without any treatment for another 8 weeks.

Light microscopic examination of the liver of GA3-treated rats revealed histological alterations in the form of marked cytoplasmic vacuolation in the hepatocytes, increased lipid droplets, pyknotic and karyolitic hepatocyte nuclei, and inflammatory cell infiltration in the portal area. Ultrastructure of the liver of GA3-treated rats confirmed the light microscopic observations. The previous changes were more severe in rats treated with $200 \mathrm{ppm}$ GA3. The toxic effects of GA3 were dose-dependent.

However, stoppage of GA3 administration for 8 weeks has resulted in some sort of regression of the previously mentioned effects; resulting in incomplete recovery of these toxic effects.

Copy Right, IJAR, 2016,. All rights reserved.

\section{Introduction:-}

Many chemicals are currently used in agriculture nowadays. One of these chemicals is the plant growth regulators where its use began in the 1930s (Fishel, 2006). Plant growth regulators (PGRs) are also known as plant hormones or phytohormones; used to regulate plant growth (Osborne and McManus, 2005).PGRs are commonly used as agricultural chemicals (Çelik et al., 2002). Gibberellic acid (GA3) is produced by a naturally-occurring fungus in large vats (Schwechheimer and Willige, 2009).

GA3 is used to increase fruit size, increase cluster size (in grapes), delay ripening of citrus fruits, speed up flowering of strawberries, and stimulate starch break down in barley (for beer making). Also, it is used to promote growth of male flowers on female plants and allows production of female-only seeds and seedless fruits (Cambell and Jane, 2002; Seiler, 2005).

The World Health Organization listed GA3 as a plant growth regulators related to pesticides. GA3 could possess risk to those professionally exposed, as well as the general population via the consumption of contaminated food products (Tuluce and Celik, 2006).

Corresponding Author:-Hanan. M. Abd El-Latief. 
Gibberellic acids (GA3) are a class of naturally occurring plant hormones, first isolated from the fungus, Fusarium, which affect plant growth, maturation and seed germination. They are a group of plant growth regulators (PGRs) that are used in agriculture to stimulate both cell division and elongation of the leaves and stems. GA3 was used in many countries including Egypt and Tunisia to increase the growth of fruits such as strawberries, grapes, date palm and some vegetables such as tomatoes, cabbages, cauliflower, pepper and olive (Chaari et al., 2006).

Although GA3 is used commonly in agriculture, its potential hazardous effects on human health were relatively unexplored. According to The U.S. Environmental Protection Agency (EPA), toxic xenobiotic chemicals are irritating to the eyes, skin and mucous membrane and since it is easily absorbed dermally, orally or by inhalation, can injure liver, kidney, muscle and brain tissues(Celik and Tuluce 2007).

The liver is the main target for the toxicity of several compounds. This is because $75 \%$ of blood coming to the liver arrives directly from gastrointestinal organs and then spleen via portal veins which bring drugs and xenobiotics in concentrated form (Lee and Senior, 2005). There are reports in support of GA3 impairment effects on the hepatic function and structure of rats (Saker et al., 2003).

Although GA3 is extensively used in Egypt and other countries, little is known about its toxic effects in mammals as well as its potential hazardous effects on human health (Saker et al., 2003; Erin et al., 2008;Amer and Hussien, 2010).The increasing use of this substance in agriculture making it as an interesting subject to investigate its possible adverse effects on the liver as one of the main target organs for different xenobiotics.

Therefore, the aim of this study was to evaluate hepatotoxic effects of GA3 in adult male albino rats for 8 weeks, and also to determine the effects of withdrawal of GA3 on the affected parameters following 8 weeks of follow up.

\section{Materials and Methods:-}

\section{Chemicals:-}

Gibaifar (5\% Gibberellic acid) supplied byAIFARAGROCHIMICASRLViaBazzano, 1216019 RoncoScrivia (Genoa) Italy.www.aifar.it

Preparation of GA3: $2 \mathrm{ml}$ and $4 \mathrm{ml}$ of $5 \%$ GA3 (equivalent to $100 \mathrm{mg}$ and $200 \mathrm{mg}$ of GA3, respectively) were diluted with tap water until $1000 \mathrm{ml}$ to obtain 100 and $200 \mathrm{ppm}$ of GA3, respectively.

\section{Experimental animals:-}

Adult male Wistar albino rats weighing 180-200 g were obtained from animal house in Medical Research center (MRC), Faculty of Medicine, Ain Shams University. The animals were housed in cages and fed ad libitum with a standard diet and provided with free access to water. They were housed in a controlled environment with room temperature and a $12 \mathrm{~h} \mathrm{light/dark} \mathrm{cycle.}$

The animals were allocated into 5 groups, each of 10 animals. The animals belonging to the first group I served as control and were received tap water. The animals belonging to group II and group III were received 100 and 200 ppm GA3daily in drinking water for eight weeks; according to Celik et al.(2006) and Troudi et al.(2012) respectively.

Since all rats have the same physiologic characters, daily water consumption of all rats was approximately $30 \pm 3 \mathrm{ml}$ during the tests. Consequently, the GA3 intake amount of each rat was about $3 \pm 0.3 \mathrm{mg}$ and $6 \pm 0.6 \mathrm{mg}$ per day for groups II and III, respectively.

Animals belonging to the group IV (recovery of low dose GA3 group) received 100 ppm of GA3 daily in drinking water for eight weeks, and then were left without any treatment for another 8 weeks. Animals belonging to the group $\mathrm{V}$ (recovery of high dose GA3 group) received $200 \mathrm{ppm}$ of GA3 daily in drinking water for eight weeks, and then were left without any treatment for another 8 weeks. On completion of the experimental period, animals from each group were sacrificed under ether anesthesia and liver were removed and fixed in the suitable fixative for histological and ultrastructural studies. 


\section{Histological studies:-}

Liver specimens from all groups were fixed in formalin and embedded in paraffin. Sections of $5 \mu \mathrm{m}$ thickness were stained with hematoxylin and eosin using standard procedures (Bancroft\& Gamble,2002). The stained sections were examined under light microscope.

\section{Ultrastructural studies:-}

Liver specimens were further processed for ultrastructural evaluation by transmission electron microscopy (TEM); the liver samples were cut into small pieces of about $1 \mathrm{~mm} 3$ and fixed in $2.5 \%$ glutaraldehyde for $24-48 \mathrm{hr}$. The specimens were then washed in $0.1 \mathrm{M}$ phosphate buffer ( $\mathrm{pH} 7.4$ ) 3-4 times for 20 min. every time and post-fixed in a buffered solution of $1 \%$ osmium tetroxide at $4^{\circ} \mathrm{C}$ for $2 \mathrm{hr}$. After dehydration in graded concentrations of ethyl alcohol, the tissue specimens were cleared in two changes of propylene oxide, and embedd in Epon resin. Semithin sections ( 1 um thick) were stained with $1 \%$ toluidine blue and examined by using a light microscope. Areas of interest were selected and the blocks were trimmed accordingly. Utrathin sections $(60-70 \mathrm{~nm})$ were cut with a diamond knife using an ultra-microtome (MT6000-X L RMC, Inc.), mounted on copper grids and double stained with uranyl acetate and lead citrate (Weakley, 1981 ). Grids were viewed and photographed using a transmission electron microscope (JEOLJEM-1200 EX 11, Japan) operated at 60-70 kV (Regional Center for Mycology and Biotechnology (RCMB), Al-Azhar University.

\section{Results:-}

\section{Light microscopic findings of the liver:-}

The liver of the control rat (Group I) exhibited the normal histological architecture. The liver is composed of hepatic lobules which are bounded by connective tissue. Each hepatic lobule is formed of hepatic cords radiating from a central vein and separated by narrow hepatic sinusoids (Fig. 1a). The angle of the hepatic lobules is separated by the portal area occupied by a branch of the hepatic vein, a branch of the hepatic artery, bile ductule and lymph vessels (Fig. 1b). The hepatocytes are polyhedral in shape with acidophilic cytoplasm and rounded centrally located nuclei. Hepatic sinusoids are lined with endothelial and Kupffer cells (Fig. 1a\&b).
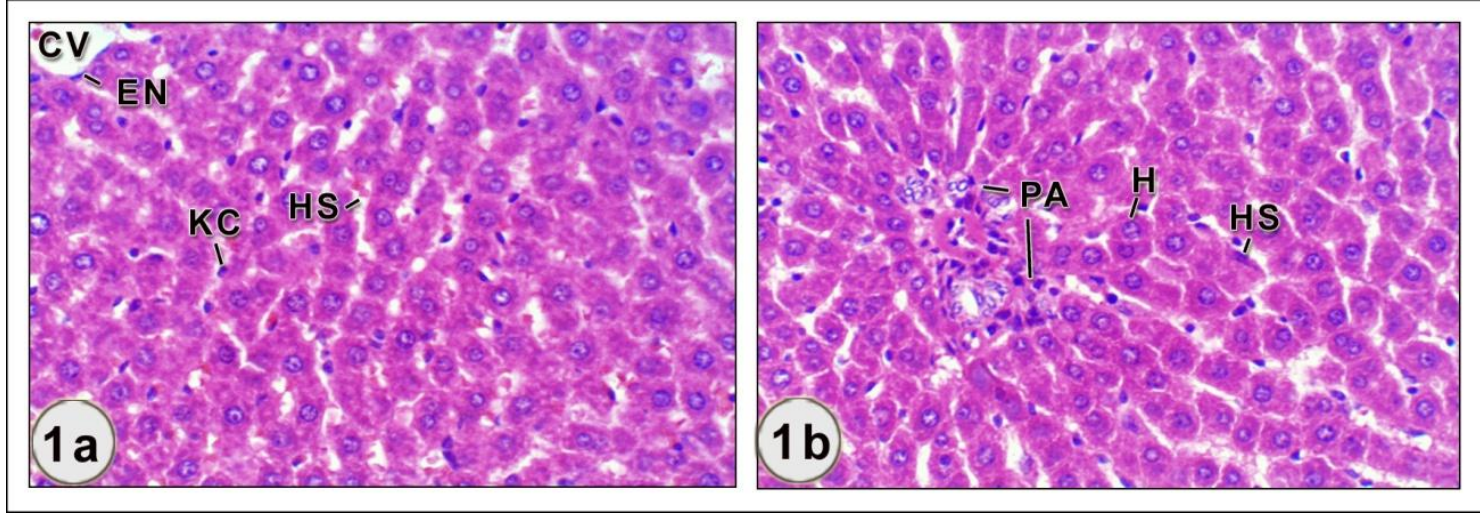

Figure 1(a-b):- Photomicrographs of liver section of control rats. (a): Showing hepatic cords radiating from the central vein $(\mathrm{CV})$ which is lined with endothelial cells $(\mathrm{EN})$, the hepatic cords are separated by the hepatic sinusoids (HS) lined by Kupffer cells (KC). (b):Showing cords of hepatocytes (H) separated by the hepatic sinusoids (HS). Note the normal hepatic portal area (PA). (H\&Ea-b X400).

H\&Estained sections of the liver of rats treated with 100 ppmGA3daily for eight weeks showed loss of the normal hepatocytes architecture, some nuclei appeared pyknotic or karyolitic, and the hepatocyte cytoplasm showed vacuolation and fatty degeneration (microvesicularsteatosis) as revealed in figures $2 \mathrm{a} \& 2 \mathrm{~b}$. Dilatation of the central veins with destruction of the endothelial lining and congested, dilated hepatic sinusoids were also seen (Fig. 2a\&b). 


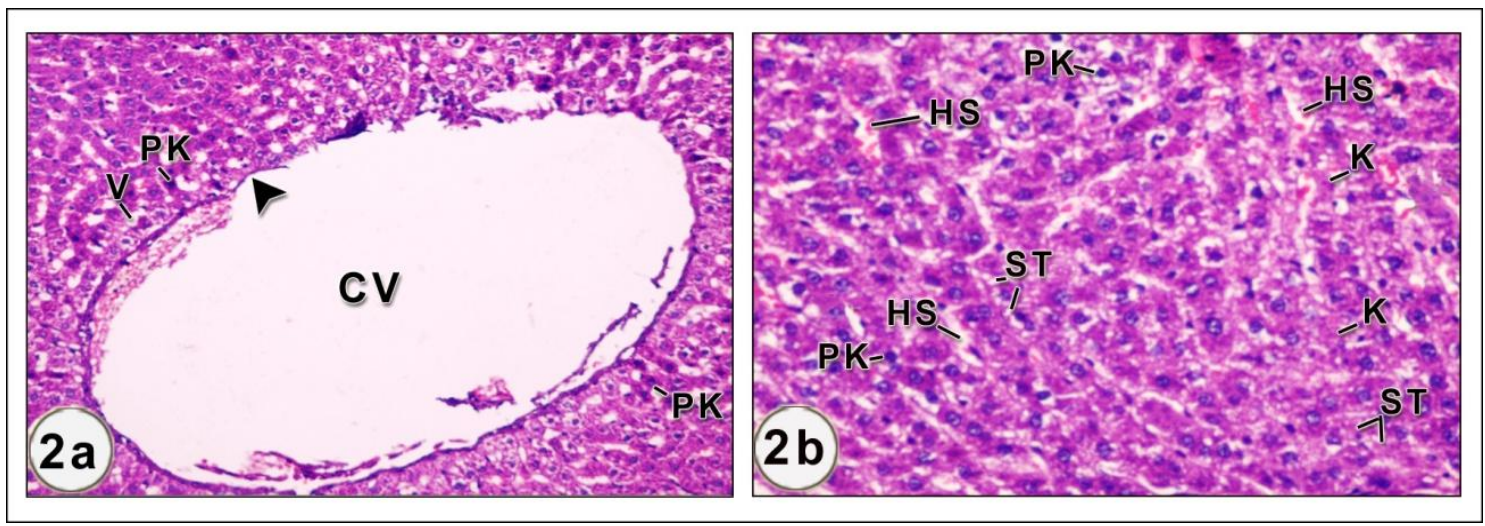

Figure2(a-b):- Photomicrographs of liver sections of rats treated with 100ppm GA3 for 8 weeks. (a):Showing dilated central vein (CV) with destruction of the endothelial lining (arrow head) and hepatocytes with vacuolated cytoplasm (V) and pyknoticnuclei(PK).(b): Showing loss of the normal architecture of the hepatocytes, some hepatocytes show microvesicularsteatosis (ST) and others show pyknotic (PK) andkaryolitic (K) nuclei. Note the dilated and congested hepatic sinusoids (HS). (H\&Ea X200; b X400).

On the other hand, liver sections from rats treated with GA3 200 ppm daily for eight weeks revealed obvious lesions in the liver tissues. Some hepatocytes revealed necrotic changes including pyknotic nuclei, other hepatocytes revealed karyolitic nuclei (Fig. 3a\&b). Microvesicularsteatosis, necroinflammatory foci in-between the hepatocytes and destruction of the hepatic strands were also observed. (Fig. 3a\&c). The hepatic sinusoids appeared congested and dilated, bile ductuleswere proliferated (Fig. 3a\& c).Dilatation, congestion of portal and central veins with aggregation of inflammatory cells were also detected (Fig. 3a, b\&d).
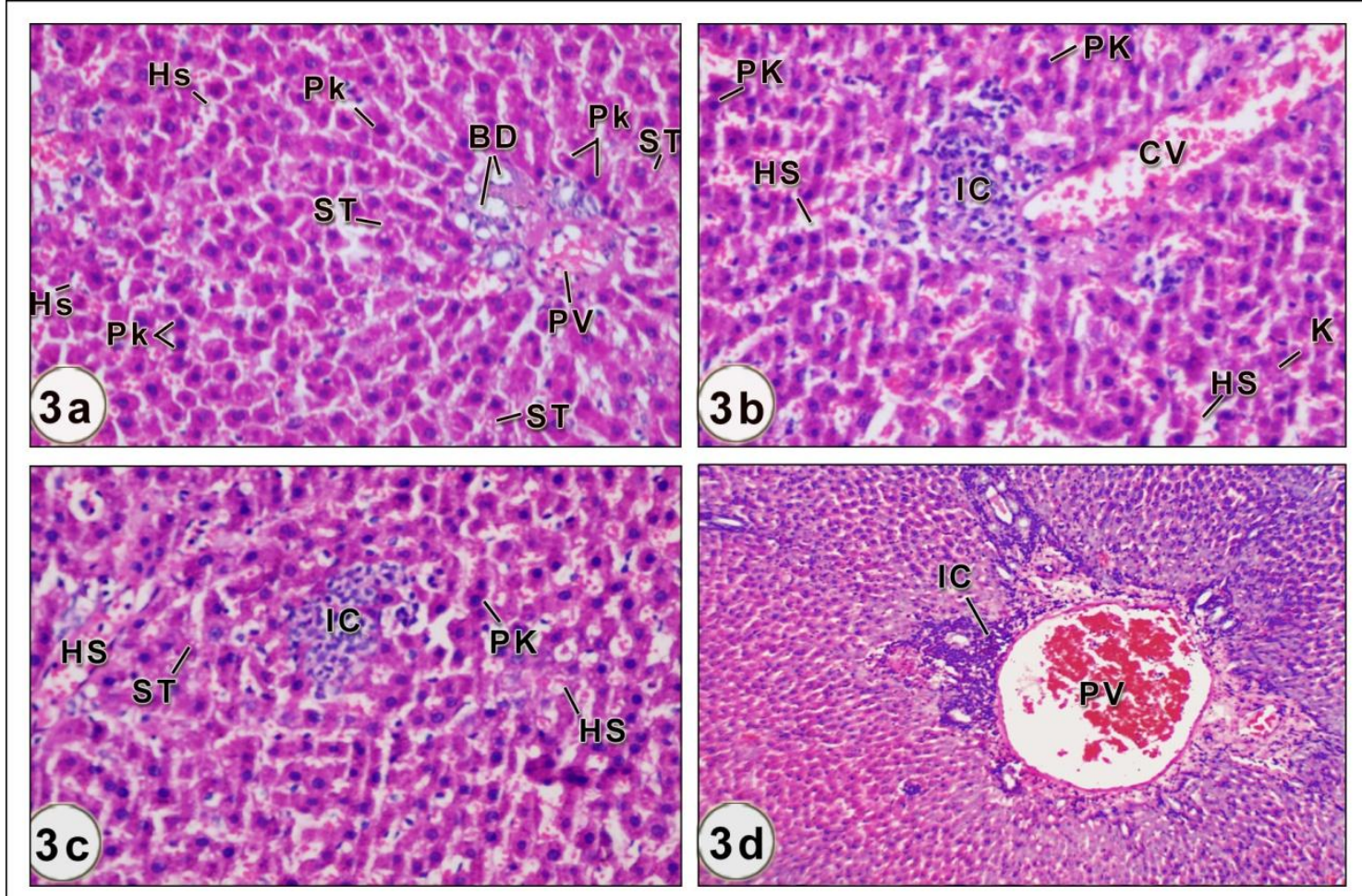

Figure 3 (a-d):- Photomicrographs of liver sections of rats treated with 200ppm GA3 for 8 weeks. (a):Showing congested portal vein (PV), proliferated bile ductules (BD), congested, dilated hepatic sinusoids (HS) andmicrovesicularsteatosis (ST) in the hepatocytes. Some hepatocytes with pyknotic nuclei (PK)were also seen. (b): Showing dilated and congested central vein (CV), lymphocytic inflammatory cells (IC) around the central vein, dilated, congested hepatic sinusoids (HS), pyknotic nuclei (PK)in some hepatocytes, other hepatocytes show 
karryolytic nuclei(K). (c): Showing destruction of the hepatic strands, necroinflammatory foci in-between the hepatocytes (IC), dilated, congested hepatic sinusoids (HS), microvesicularsteatosis (ST) in some hepatocytes and pyknotic hepatocyte nuclei(PK). (d):Showing dilated, congested portal vein (CV), and severe inflammatory cell (IC) infiltration in the portal area. (H\&E a, b \&c X400; d X100).

Liver sections fromgroup IVrats revealed preserved radially arranged hepatocytes around the central vein with marked recovery of degenerative changes in the hepatocytes (Fig. 4). Liver sections from rats from the recovery group $\mathrm{V}$ revealed partial restoration of the normal architecture of the liver tissue. Most of hepatocytes showed ground glass appearance. However, few vacuoles and lipid droplets were observed in the hepatocyte cytoplasm (Fig. $5)$.
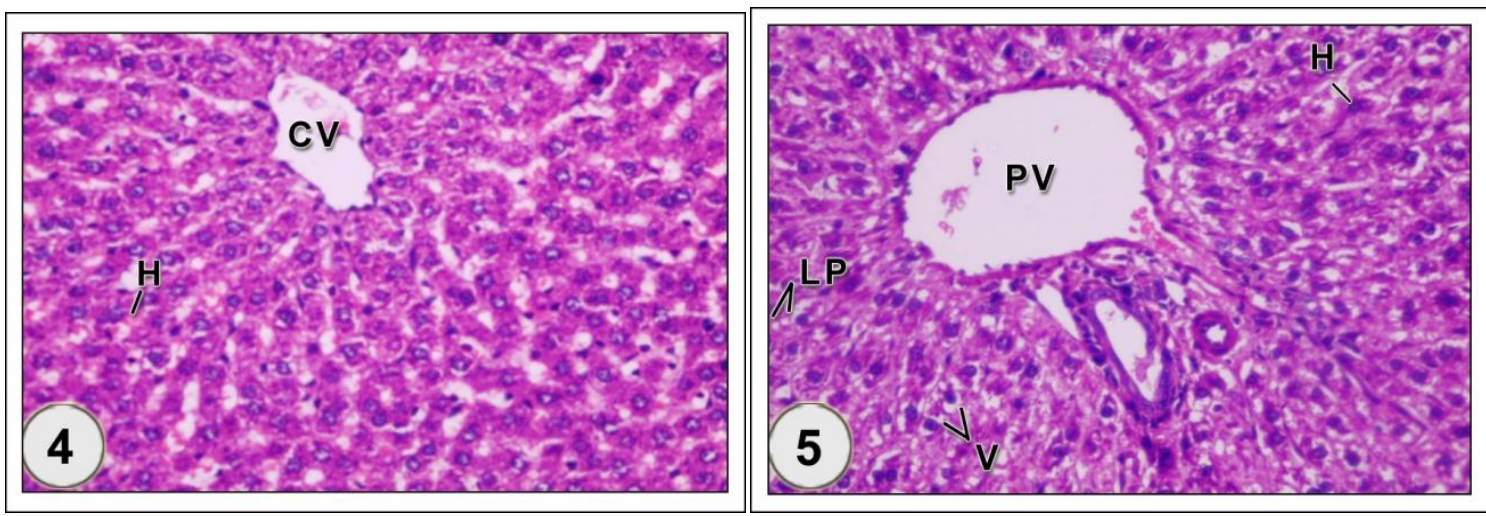

Figure 4:- Photomicrograph of a liver section of a rat treated with 100ppm GA3 for 8 weeks then left without treatment for another 8 weeks (recovery of low dose GA3) showing restoration of the normal appearance and structure of hepatocytes $(\mathrm{H})$ and central vein $(\mathrm{CV})$. (H\&E X 400).

Figure5:- Photomicrograph of a liver section of a rat treated with 200 ppm GA3 for 8 weeks then left without treatment for another 8 weeks (recovery of high dose GA3) showing dilated portal vein (PV), few lipid droplets (LP), mild cytoplasmic vacuolization (V) and ground glass appearance of hepatocytes (H). (H\&EX 400).

\section{Ultrastructural findings of the liver:-}

Ultrastructural examination of the liver of the control rats revealed that each hepatocyte exhibited a rounded large central nucleus surrounded by an envelope of 2 membranes interrupted by nuclear pores; the nucleus possessed light euchromatin and dark heterochromatin. The cytoplasm of the hepatocytes contained numerous mitochondria, rough endoplasmic reticulum, Golgi apparatus and lysosomes. There are also glycogen inclusions and lipid droplets (Fig. $6 a \& b)$.
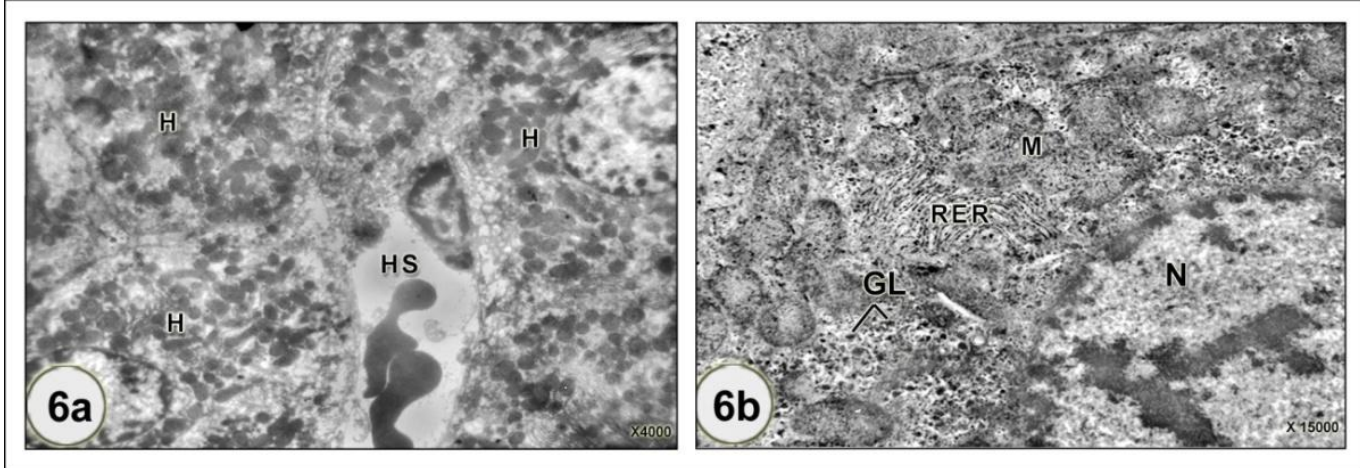

Figure 6 (a-b):- Transmission electron micrographs of liver of control rats. (a):Showing hepatocytes $(\mathrm{H})$ and hepatic sinusoids (HS). (b):Showing part of hepatocyte nucleus (N), the hepatocyte cytoplasm contains mitochondria (M), rough endoplasmic reticulum (RER) and glycogen particles (GL). (TEM a X4000; b X15000).

Examination of the hepatocytes of animals of Group II showed ultrastructural alterations. The nuclei of some hepatocytes showed pyknosis, other nuclei appeared with ill-defined nuclear membrane (Fig. 7a\&b). The cytoplasm 
of the hepatocytes showed polymorphic electron-dense mitochondria, partial fragmentation of the rough endoplasmic reticulum, proliferation and dilatation of the smooth endoplasmic reticulum and many lipid droplets (Fig. 7a \&b).

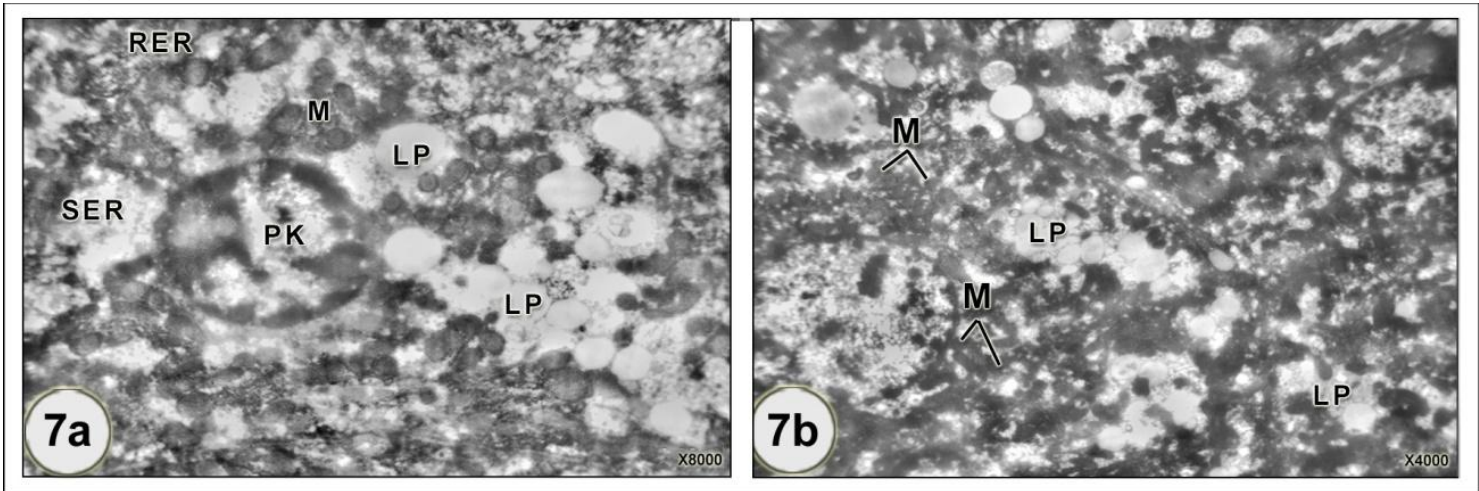

Figure7 (a-b):- Transmission electron micrographs of liver of rats treated with 100 ppm GA3 for 8 weeks. (a):Showing hepatocyte contains pyknotic nucleus (PK)and abnormal cytoplasm with electron dense small-sized mitochondria (M), many lipid droplets (LP), fragmented rough endoplasmic reticulum (RER) and dilated smooth endoplasmic reticulum (SER). (b):Showing hepatocytes contain increased lipid droplets (LP)and electron-dense damaged mitochondria (M). (TEM a X8000; b X4000).

Ultrastructural examination of the liver sections from rats of Group III demonstrated obvious lesions. The nuclei of some hepatocytes showed pyknosis with ill-defined nuclear envelope (Fig. 8b\&c). Polymorphic fused mitochondrial, proliferation and dilatation of smooth endoplasmic reticulum, fragmentation of rough endoplasmic reticulum and massive cytoplasmic lipid droplets were also seen (Fig. 8a-d). Dilated hepatic sinusoids with erosion of the plasma membrane of the endothelial lining cell and partial to total loss of hepatocytes microvilli were detected (Fig. 8a\&b). Dilated bile canaliculi deprived of microvilli were also noted (Fig. 8c).

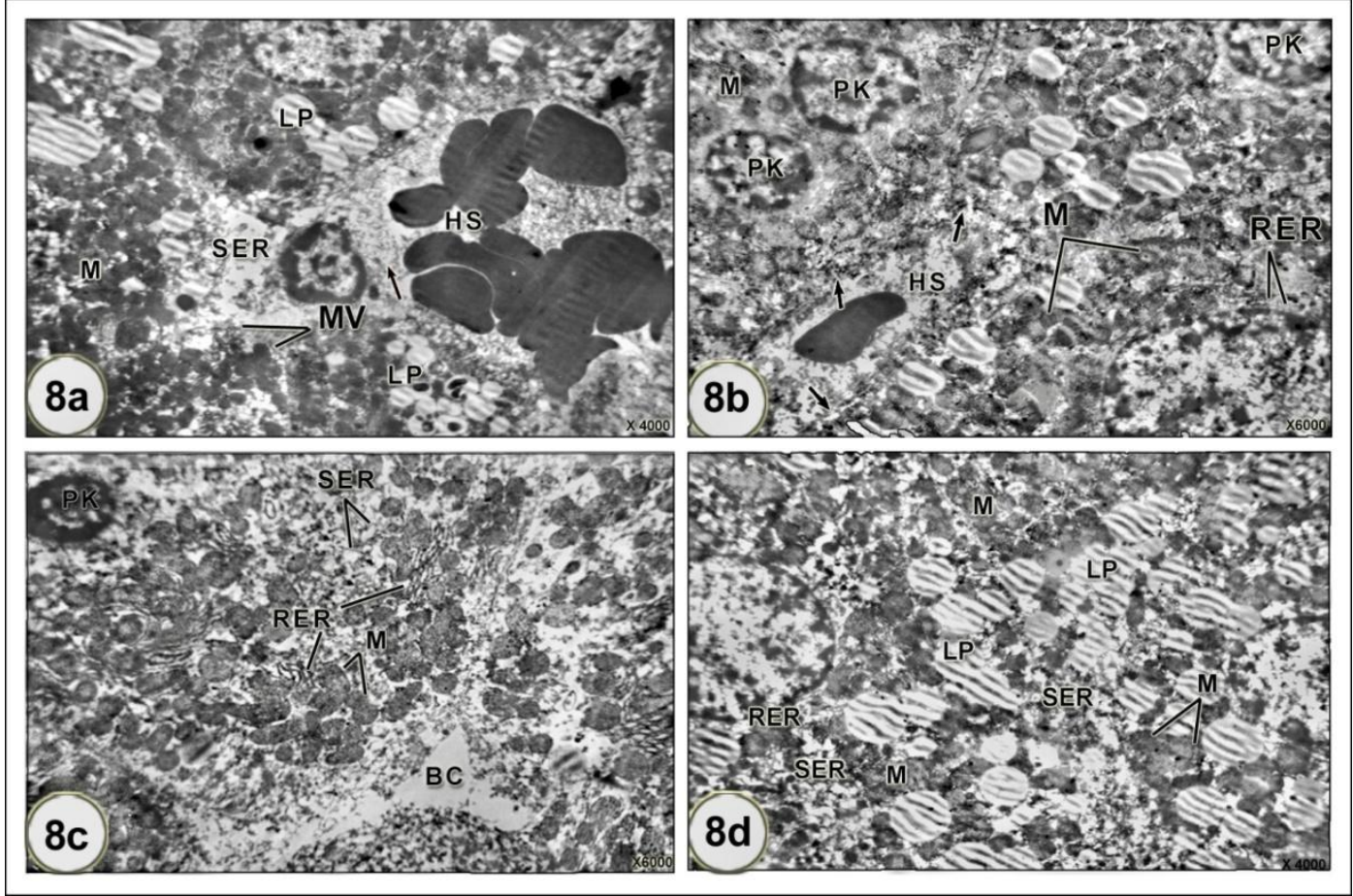

Figure8 (a-d):- Transmission electron micrographs of liver of rats treated with 200 ppm GA3 for 8 weeks. (a):Showing hepatocytes and hepatic sinusoid, the hepatocytes cytoplasm contains proliferated smooth endoplasmic reticulum (SER) abnormal mitochondria (M) and increased lipid droplets (LP). The hepatic sinusoid (HS) appeared dilated, congested with erosion of the plasma membrane of the endothelial lining cell (arrow). Notice the destruction of the hepatocytes microvilli (MV) projecting in the space of Disse. (b):Showing hepatocytes and 
hepatic sinusoid (HS), the hepatocyte revealedpyknotic nuclei(PK), coalescence of the mitochondria (M) and short stacks of rough endoplasmic reticulum (RER). Hepatic sinusoid (HS) appear with destructed hepatocytes microvilli (arrow). (c):Showing hepatocytes and bile canaliculus (BC), with widened lumen and complete loss of microvilli. The hepatocytes showed pyknotic nuclei (PK), fragmented rough endoplasmic reticulum cisternae (RER), proliferated smooth endoplasmic reticulum (SER) and polymorphic mitochondria (M). (d):Showing high aggregation of lipid droplets (LP) in the hepatocyte cytoplasm giving the cytoplasm a rather vacuolated appearance, proliferated smooth endoplasmic reticulum (SER), fragmented rough endoplasmic reticulum (RER) and fused mitochondria(M). (TEM a X 4000; b \& c X6000; d X4000).

The electron microscopic study of the recovery group IV showed marked improvement of the hepatocytes. The hepatocytes almost restored their normal appearance and structure; their nuclei appeared vesicular with homogenous chromatin distribution and peripheral nucleolus (Fig. 9). The mitochondria, rough endoplasmic reticulum and glycogen almost retained their normal appearance (Fig. 9).

However, ultrastructure examination of the liversections of rats from group $\mathrm{V}$ revealed partial restoration of the hepatocyte appearance as their nuclei appeared vesicular and the cytoplasm contained numerous mitochondria; some mitochondria showed electron-dense matrix. Rough endoplasmic reticulum and glycogen particles almost retained their normal appearance (Fig. 10). The hepatocyte cytoplasm although displayed few lipid droplets; and some hepatic sinusoids appeared with swollen endothelial cell were also noted (Fig. 10).
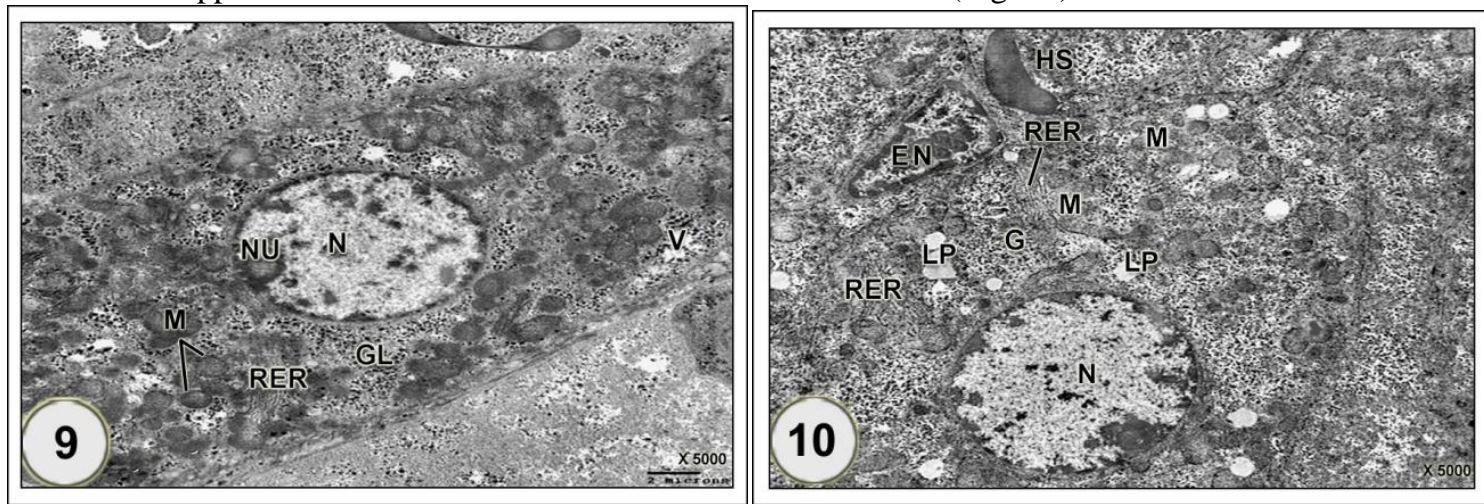

Figure 9:-A transmission electron micrograph of a liver from a rat treated with 100 ppm GA3 for 8 weeks then left without treatment for another 8 weeks (recovery of low dose GA3)showing good recovery of the hepatocyte. The rough endoplasmic reticulum (RER), mitochondria (M) and glycogen particles (GL) almost return the normal appearance. Large centric euchromatic nucleus $(\mathrm{N})$ with peripheral nucleolus $(\mathrm{Nu})$ is also noticed. The hepatocyte cytoplasm with few vacuoles (V) is still seen. (TEM X5000).

Figure 10:-A transmission electron micrograph of a liver of a rat treated with 200 ppm GA3 for 8 weeks then left without treatment for another 8 weeks (recovery of high dose GA3)showing partial restoration of the hepatocyte organelles, mitochondria (M), rough endoplasmic reticulum (RER) and glycogen inclusions (GL) appear with nearly normal appearance. Notice the presence of few lipid droplets (LP). The hepatic sinusoid (HS) with swollen endothelial cell (EN) was also noted. (TEMX5000).

\section{Discussion:-}

It is well known that the liver is the first target organ in toxicological prospects regarding its role in detoxification, biotransformation and excretion of xenobiotic. After enteric uptake of injurious materials, it is the first organ to be exposed to such hazards via the portal circulation (Katzung, 1990).

Results obtained in the present study showed that gibberellic acid induced many histopathological changes in the liver of rats. Similarly, Abdelhamid et al. (1994) reported that feeding chickens with GA3 led to numerous histological lesions in different organs including liver and that two-week withdrawal period did not ameliorate its effect.

Light microscopic examination of liver sections of GA3 treated rats for 8 weeks showed cytoplasmic vacuolization of the hepatocytes, fatty degeneration (microvesicularsteatosis) with inflammatory cells in between the hepatocytes 
and pyknotic hepatocyte nuclei. The hepatic sinusoids appeared congested, dilated. Also, dilated central and portal veins with many inflammatory cells were also noticed. These findings come in accordance with the findings of Sakr et al. (2003) who reported that GA3 induced histopathological changes in the liver such as cytoplasmic vacuolization of the hepatocytes with pyknotic nuclei, congested blood vessels and inflammatory leucocytic infiltrations. The intrahepatic, central and portal veins and the hepatic sinusoids were noticed in the present work to undergo marked injury indicated by dilatation of their lumina and aggregation of blood cells in some of them. The above mentioned observation come in agreement with that obtained by Troudi et al. (2010) who stated that in rats exposed to GA3, the intrahepatic blood vessels, central and portal veins were congested and their lining epithelia were eroded. Authors also noticed massive inflammatory cellular infiltrations in several areas of the lobules and stated that such injuries were more obvious in the peripheral lobular zones than the pericentrally located ones. The results of the present work paralleled to Hussien et al. (2011) who revealed, mild piecemeal necrosis of hepatocytes, pyknotic nuclei and inflammatory cellular infiltrates in rats received 75 ppm of GA3 daily in drinking water for six weeks.. The histological alterations observed in the present work come in accordance with those obtained by Hassan et al. (2013)who reported that the liver of animals treated with GA3 showed dilated central vein with degeneration of endothelial cells, vacuolation of the hepatocyte cytoplasm, congested sinusoids and severe lymphocytic infiltration in the portal region. Among the pathological symptoms observed in the present work was the remarkable abundance of leucocytic infiltrations in the liver of GA3-treated animals. Mohamed (2015) also revealed vacuolated hepatocytes with pyknotic nuclei and inflammatory cellular infiltrations. The central and portal veins appeared congested and dilated with dilated blood sinusoids in liver of animals treated with GA3.

These leucocytic infiltrations were considered as a prominent response of the body tissue facing any injurious impacts (Abdel-Rahaman and Zaki, 1992).In the current study, hepatocytes showed severe cytoplasmic vacuolization. The cytoplasmic vacuolation had been subjected to wide speculations by many investigators. Zhang and Wang (1984) correlated the vacuolar degenerative changes with the marked disturbances which take place in lipid inclusions as a result of injurious treatments. Sherlock and Dooley (2002) demonstrated that cytoplasmic swelling and vacuolization are one of the most important primary responses to all forms of cell injury. They occurred due to increased permeability of cell membranes leading to an increase of intracellular water. As water sufficiently accumulates within the cell, it produces cytoplasmic vacuolization.

One of the characteristic features observed in the present work was the remarkable abundance of lipid droplets in the hepatocyte cytoplasm. The increase in fat was explained by Mori (1983) who stated that increased lipid in the hepatocytes after exposure to drugs or toxins could be due to impaired synthesis of lipoproteins or due to the abnormal transport of lipoproteins via Golgi apparatus and its secretory vacuoles. Lipid accumulation in the hepatocytes is well known as a pathological change that occurs in the cells under a variety of deleterious conditions and could be due to an alteration in the metabolism of the hepatic cells (Wheater et al., 1990). Dilatation of hepatic sinusoids, presently, observed was also explained by Klatskin and Conn (1993) who suggested that dilatation of the blood sinusoids would be due to the direct toxic effect of the toxin on the blood sinusoids leading to their dilatation.

In the present work, ultrastructural findings in liver of GA3-treated rats revealed polymorphic, atrophied, electrondense mitochondria with, proliferation of smooth endoplasmic reticulum, vesiculation and fragmentation of the rough endoplasmic reticulum, increased lipid droplets, cytoplasmic vacuoles and loss or destruction of hepatocyte microvilli. The above mentioned observations are in agreement with the results obtained by Abd El Maksoud et al. (1996) who recorded that oral administration of GA3 revealed significant ultrastructural changes in the liver cells in the form of appearance of large areas of rarified cytoplasm with disappearance of cellular organelles. In this concern, Kendry and Laszlo (1975)concluded that the alteration of the rough endoplasmic reticulum constitutes the main adverse effect of drug and toxins affecting the liver due to its important role in protein synthesis. Mohamed (2015) also revealed that in the liver of male albino rats treated with GA3 ultrastructural changes manifested as rarified cytoplasm, degenerated vacuolated mitochondria, dilated rough endoplasmic reticulum, and activated Kupffer cells.

The interpretation of the cytoplasmic swelling and vacuolations had been subjected to wide speculations by many investigators. Sherlock and Dooly (2002) demonstrated that cytoplasmic swelling and vacuolization are one of the most important primary responses to all forms of cell injury. They occurred due to increased permeability of cell membranes leading to an increase of intracellular water. As water sufficiently accumulates within the cell, it produces cytoplasmic vacuolization. 
Many earlier studies disclosed that mitochondrial dysfunction contributed to apoptosis via the production of reactive oxygen species (Mignotte and Vayssiere, 1998). For interpretation of the mechanism of mitochondrial swelling reported in this study, Jaeschke et al. (2002) mentioned that oxidative stress had contributed to the opening of the mitochondrial permeability transition pore (PTP) which led to the formation of a high-conductance channel; in the inner mitochondrial membrane, and led to mitochondrial swelling and subsequent release of cytochrome $\mathrm{c}$ from the intermembrane space. PTP opening appears to be associated with apoptosis or necrosis according to the presence or deficiency of ATP (Lemasters, 2002).

Also, Attia et al. (2014) revealed that the appearance of many cytoplasmic changes might reflect the association between GA3 and oxidative stress. As regarding the follow up period in this study, H\&E stained liver sections of the recovery groups showed marked and partial recovery in groups IV and V, respectively.

The ground glass appearance of hepatocytes detected in the recovery period of this study coincides with Droge (2002) who mentioned that the glass appearance reflect adaptation of cells upon recovery. Also, these results agree with Kimball (2008) who stated that the ground glass appearance of liver cells was considered as adaptive mechanism and occurred as a result of increased synthesis of cellular organelles such as mitochondria and RER probably to increase function of individual hepatocytes.

Abundant SER in the recovery period could be also explained by Benedetti (2005) who stated that it takes part in the synthesis of phospholipids for building of cell membrane and membranes of cell organelles.

On the other hand the increased RER was explained by Kimball (2008) who mentioned that RER had a very important role in the synthesis and packaging of proteins. Some of these proteins might be used by the cell to synthesize membrane, and other cell organelles. This was considered as a structural-functional response to enable cells to release the oxidative stress secondary to GA3 toxicity.Abdelhamid et al. (1994) reported that feeding 2weeks old broiler chicks on gibberellic acid (GA3)- containing diets $(0,1,5,25$ and $125 \mathrm{ppm})$ for 3-weeks led to numerous histological lesions in different organs. Two-week withdrawal period did not ameliorate the negative effects of GA3. Also, Abou-zeid and Abd-Ellah (2015) suggested that the toxic effects of GA3 were dosedependent. While, after GA3 stoppage, 8 weeks period of follow up showed incomplete recovery of these toxic effects. So, gibberellic acid should be used cautionary.

Mohamed (2015) revealed that GA3 has histological and immunohistochemical effects on albino rat liver cells and that green tea has a minimal protective effect.

\section{Conclusion:-}

From the aforementioned results and the previously mentioned results it can conclude that GA3 was a potent prooxidant that induced a significant hepatotoxicity in adult male albino rats following 8 weeks of daily exposure. On the other hand, 8 weeks period of follow up was insufficient for complete recovery of these toxic effects.

\section{Recommendations:-}

1. Gibberellic acid (GA3) usage should be under strict control.

2. More studies are needed to explore other hazardous effects of GA3 on other body systems and organs.

3. Other studies with prolonged periods of GA3 administration are recommended to learn more about its toxic effects.

\section{Conflict of interest:-}

The author declares that there are no conflicts of interest.

\section{References:-}

1. Abd El Maksoud SA, Abd El Maksoud N, Abd El Hamid NA(1996). Chronic toxic effect of plant growth promoting hormone, gibberellic acid, on the liver cells of adult male albino rat.Assut Med J 20: 87-103.

2. Abdelhamid AM, Dorra TM, Ali MA ,Abou-Egla EH (1994).Effect of gibberellic acid on broiler chickens performance and some metabolic parameters. Arch Tierernahr 46(3):269-276.

3. Abdel-Rahaman M, ZakiTZ(1992). Cytotoxic action of malathion on renal and hepatic tissues of mice. J Egypt Germ SocZool 8: 105-114. 
4. Abou-zeid NR, Abd-Ellah H F(2015). Neurotoxic Effects of Gibberellic Acid (GA3) and its Withdrawal in Adult Male Albino Rats: A Light and Electron Microscopic Study . Global Journal of Pharmacology 9 (3): $222-$ 233.

5. Amer M G,Wafaa F, Hussien, WF( 2010). Influence of Gibberellic Acid (GA3) on Renal Cortex of Adult Male Albino.

6. Attia AA, kheirallahNA, Khalifa SA( 2014). Biochemical and ultrastructural studies of the effect of alprazolam as an anxiolytic drug on the cerebellum of adult male mice. J App Pharm Sci 4 (1):74-83.

7. Bancroft JD,GambleM (2002). Theory and practice of histological techniques. 5th ed. London: Churchill Livingstone.

8. Benedetti A,2005. Endoplasmic reticulum: a metabolic compartment. FEBS Letters, 580:2160-2165.

9. Cambell NA, Jane B(2002). Biology, 6th ed. San Francisco., Benjamin Cumming.145-155.

10. Çelik İ, Özbek H, Tuluce Y( 2002). Effects of subchronic treatment of some plant growth regulators on serum enzyme levels in rats. Turk J Biol 26:73-76.

11. Celik I, Tuluce Y, Isik I(2006). Influence of subacute treatment of some plant growth regulators on serum marker enzymes and erythrocyte and tissue antioxidant defense and lipid peroxidation in rats. $J$ BiochemMolToxicol. 20(4):174-182.

12. Celik I, TuluceY, Isik I(2007). Evalution of toxicity of abcisic acid and gibberellic acid in rats: 50 days drinking water study. J Enzyme InhibMedChem. 2 (22):219-226.

13. Chaari RA, Maalej M, Ouled M S , Drira N( 2006). In vitro vegetative growth and flowering of olive tree in response to GA3 treatment. Afr J Biotech 5(22):2097-2302.

14. DrogeW( 2002).Free radical in the physiological control of cell function. Physiol Rev 82(1):47-95.

15. Erin E, Afacan B, Ersony Y, Ercan F, Blaci MK( 2008). Gibberellic acid, a plant growth regulator increase mast cell requirement and alters substance $\mathrm{P}$ levels. Tox 245: 75-81.

16. Fishel FM(2006). Gibberellins Agronomy Department, Florida Cooperative Extention Service Institute of Food and Agricultural Sciences University of Florida USA.

17. HassanHA, Al-Rawi MM( 2013). Grape seeds proanthocyanidin extract as a hepatic-reno-protective agent against gibberellic acid induced oxidative stress and cellular alterations. Cytotechnology 65(4):567-576.

18. Hussein WF,FarahatFY, Abass M, Shehata AS( 2011). Hepatotoxic potential of gibberellic acid (GA3) in adult male rats. Life Science Journal 8(3):373-383.

19. Jaeschke H, Gores GJ, CederbaumAI,Hinson JA, Pessayre, D, LemastersJJ(2002). Mechanism of hepatoxicityToxicolSci 56: 76-66.

20. Katzung BG(1990). Basic and clinical pharmacology 3rd ed Appleton and langconnreticut U.S.A.

21. KendryG, LaszloB(1975). "Morphologic spectrum of drug-induced liver damage.Light and electron microscopic study". Love Arch Mag 28, pp 269.

22. KimballJW(2008).Growthregulatinghormones.http://users.rcn.com/jkimball.ultranet/biologypages

23. Klatskin G, ConnHO(1993). Histopathology of the liver Vol.I\& II. Oxford University Press New York.

24. Lee WM, Senior JR(2005). Recognizing drug-induced liver injury: Current problems, possible solutions. J ToxicolPathol 33 (1):155-164.

25. LemastersJJ(2002). Mechanisms of hepatotoxicity.ToxicolSci 65: 166-167.

26. Mignotte B, VayssiereJL( 1998). Mitochondria and apoptosis.Eur J Biochem 15:252:1-15.

27. Mohamed S T( 2015). Histological and immunohistochemical study on the effect of gibberellic acid on the liver of adult male albino rats and the possible protective role of green tea. Egyptian Journal of Histology 38(2):317331.

28. Mori M(1983). Ultrastructural changes of hepatocyte organelles induced by chemicals and their relation of fat accumulation in the liver. Actapathol Jpn33 (5): 911-922.

29. Osborne DJ, McManusMT(2005).Hormones Signals and Target Cells in Plant Development. 1st edVol 1 Cambridge University Press pp 266.

30. SakrSA,OkdahYA, EL- AbdSF(2003). Gibberellin A3 induced histological and histochemical alterations in the liver of albino rats. Science Asia 29:327-331.

31. SchwechheimerC, WilligeBC(2009). Shedding light on gibberellic acid signaling CurrOpin Plant Biol 12(1):5762.

32. SeilerSE(2005). Plant growth regulation. In: Forest Biology Textbook chp5 pp.150.

33. SherlockS,DooleyJ(2002).Diseases of the Liver and Biliary System 9th ed Oxford Boston Blackwell Scientific Publications pp. 114-131. 
34. TroudiA, BouazizH, SoudaniN, Ben Amara I,BoudawaraT, TouzaniH,LyoussiB, ZeghalN(2012). Neurotoxicity and oxidative stress induced by gibberellic acid in rats during late pregnancy and early postnatal periods: Biochemical and histological changes. ExpToxicolPathol. 64(6): 583-590.

35. TroudiA, Samet AM, Zeghal N(2010). Hepatotoxicity induced by gibberellic acid in adult rats and their progeny. Ex ToxicolPathol. 62(6): 637-642.

36. TuluceY,Celik I( 2006). Influence of subacute and subchronic treatment of abcisic acid and gibberellic acid on serum marker enzymes and erythrocyte and tissue antioxidant defense systems and lipid peroxidation in rats. Pest Biochem Physiol. 86:85-92.

37. WeakleyBS(1981). A Beginner's Handbook in Biological Transmission Electron Microscopy 2nd ed. Churchill Livingstone London Chap 2: pp. 24-25.

38. WheaterPR,BurkittH.G,StevensA, Lowe J S(1990)."Basic Histopathology”.Churchill Livingstone, London and New York.

39. ZhangLY, WangC.X( 1994). Histopathological and histochemical studies on the oxic effect of brodifacoum in mouse liver.Zhongguoyixuekexueyuanxuebao.ActaAcademiaeMedicinaeSinicae 6(5):386-38. 